\title{
Global metabolite profiling based on GC-MS updates and LC-MS/MS analyses in ABF3-overexpressing soybean with enhanced drought tolerance
}

\author{
Kyong-Hee Nam ${ }^{1,2}$, Do Young Kim², Hye Jin Kim², In-Soon Pack², Hye Jeong Kim³ , Young Soo Chung ${ }^{3}$, \\ Soo Young Kim ${ }^{4}$ and Chang-Gi Kim² ${ }^{2^{*}}$
}

\begin{abstract}
Abscisic acid (ABA) is a phytohormone that plays an important role in the adaptive responses to abiotic stresses. We examined the metabolic changes in transgenic soybean that over-expressed Arabidopsis ABA responsive elementbinding factor 3 (ABF3), which participates in drought tolerance. Transgenic and non-transgenic plants were exposed to a water deficit, and their metabolic differences were verified by untargeted GC-MS and LC-MS/MS analyses. A total of 64 and 476 primary and secondary metabolites from leaf extracts were identified based on GC-MS and LC-MS/MS platforms, respectively. Principal component analysis derived from both GC-MS and LC-MS/MS data showed a clearly greater separation in the metabolite profiles among three different degrees of drought stress. However, no discrimination of metabolites between transgenic and non-transgenic plants was apparent. Furthermore, except for some free amino acids, quantitative differences in relative levels of those metabolites were less than $50 \%$ between genotypes. These results suggest that, during periods of drought, overexpression of ABF3 in transgenic soybean might result in a negligible variance in primary and secondary metabolism when compared with its non-transgenic counterpart.
\end{abstract}

Keywords: Abscisic acid-responsive element-binding factor 3, Abscisic acid, Drought, Gas chromatography-mass spectrometry, Liquid chromatography-tandem mass spectrometry, Metabolite, Soybean (Glycine max)

\section{Introduction}

The phytohormone abscisic acid (ABA) plays a crucial role in promoting tolerance to abiotic stresses such as drought, low temperature, and high salinity [1]. During the vegetative growth phase, ABA mediates adaptive responses to adverse abiotic conditions by stimulating stomatal closure in guard cells, changing the expression of numerous stress-responsive genes, and promoting the accumulation of compatible solutes [2]. These responses enhance plant growth and survival under stress conditions $[3,4]$.

Members of a specific subfamily of basic leucine zipper transcription factors (TFs) that recognize ABA-responsive elements (ABREs), known as ABRE-binding factors

\footnotetext{
${ }^{*}$ Correspondence: cgkim@kribb.re.kr

${ }^{2}$ Bio-Evaluation Center, Korea Research Institute of Bioscience \&

Biotechnology, Cheongju 28116, Republic of Korea

Full list of author information is available at the end of the article
}

(ABFs), are the predominant TFs in the ABA-dependent signaling pathway $[5,6]$. Their overexpression confers several phenotypes, such as hypersensitivity to ABA and sugar, reduced transpiration, greater drought tolerance, and altered expression of ABA-/stress-responsive genes [7]. These ABFs modulate stomatal closure, which then reduces water losses and increases the survival rate for transgenic plants under stress conditions. In particular, overexpression of $A B F 3$ leads to significantly higher drought tolerance in several species [8-12].

Although the general effects of $A B F 3$ on diverse abiotic stresses are known, the corresponding metabolic responses in $A B F 3$-overexpressing plants under actual stress conditions are not well understood. Metabolic analysis is a key tool for clarifying information about downstream genetic responses. This approach has already revealed a more comprehensive and holistic framework for presenting possible compositional 
differences between transgenic and conventionally bred plants [13, 14]. Furthermore, it has helped to identify unfavorable metabolites, including toxic compounds, or any unintended metabolic changes due to such genetic modifications [15-17].

Soybean (Glycine max) is an important food source that contains not only beneficial proteins, oils, and bioactive compounds, but also antinutrients such as lectin, phytic acid, raffinose, and stachyose. It is also one of the world's most widespread transgenic crops, accounting for $50 \%$ of all the land area used to grow such plants globally [18]. However, most transgenic soybeans have been developed to be resistant to herbicides and insects or to change the composition of fatty acids, rather than for tolerance to abiotic stress. Kim et al. [11] have recently reported that the introduction of $A B F 3$ induces tolerance to drought and salt stresses in transgenic soybean. They further suggested that the overexpression of this gene in soybean is closely correlated with the promotion of stomatal closure, maintenance of normal chlorophyll concentrations, and prevention of damage to cell membranes. Here, we investigated the global metabolic responses of transgenic soybean over-expressing Arabidopsis ABF3 during a drought period. Both transgenic and non-transgenic plants were cultivated under water-stress conditions in a field rainout shelter. We then performed analyses via gas chromatography-mass spectrometry (GC-MS) and liquid chromatography-tandem mass spectrometry (LC-MS/ MS) with leaf samples to obtain untargeted metabolite profiles.

\section{Materials and methods}

\section{Plant material and growth conditions}

Soybean line ' \#2' that over-expresses $A B F 3$ was produced via Agrobacterium tumefaciens-mediated transformation according to the method described by Kim et al. [11]. Seeds of this transgenic line and the 'Kwangan' wild type (WT) were sown on plastic trays filled with horticultural soil (Nongwoobio Co., Suwon, Korea) and then grown in a greenhouse maintained with day/night temperatures of $28 \pm 2{ }^{\circ} \mathrm{C} / 27 \pm 2{ }^{\circ} \mathrm{C}$ and long-day conditions (15 h light/9 h dark). Two-week-old plants were transplanted into six replicated plots (56 plants per plot) in the rainout shelter located in an experimental field at the Korea Research Institute of Bioscience and Biotechnology, Republic of Korea. The experiment utilized a randomized complete block design, and plants were spaced at $0.6 \mathrm{~m} \times 0.15 \mathrm{~m}$. Prior to transplanting, all plots were fertilized with N, P, and $\mathrm{K}$ at rates of 5.4, 3.0, and $10.7 \mathrm{~kg}$ per 10 acres, respectively. Water was supplied via sub-surface drip irrigation at 2-d intervals.

\section{Drought stress treatment}

Drought conditions were induced by suspending irrigation two weeks after transplanting. The severity of stress was assessed daily based on soil water content and visual examination of the leaflets. Soil water content was measured at 30-min intervals through three EasyAG soil moisture sensors (Sentek, Stepney, South Australia, Australia) installed in the soil at depths of 10,20,30,40, and $50 \mathrm{~cm}$, as we have described previously [19]. Before the stress treatment began, the initial soil water content was $13.4 \%$. Thereafter, values that were $0 \%, 50 \%$, and $60 \%$ lower than the initial content were chosen to represent non-, mild-, and moderate-drought conditions, which corresponded to 0,4 , and 9 weeks, respectively, of continuous drought (Fig. 1). Leaves from plants under mild-drought conditions began to show wilting while those under moderatedrought conditions were rolled downward and chlorotic.

\section{Metabolite extraction and GC-MS analysis}

Metabolite analysis via GC-MS was conducted as we have described previously [20]. At Weeks 0 (nondrought), 4 (mild-drought), and 9 (moderate-drought), two plants from each of the six replicated plots were collected and the samples were pooled from each plot to analyze metabolites. One $\mu \mathrm{L}$ of a derivatized sample was injected at a split ratio of 10:1 into a GC-MS (TRACE 1310/ISQ-LT; Thermo Scientific, Waltham, MA, USA) equipped with a VF-5 MS capillary column $(6 \mathrm{~m} \times$ $0.25 \mathrm{~mm}$ i.d., $0.25 \mathrm{~mm}$ particle size; J\&W Scientific, Folsom, CA, USA), at a constant flow of $1.2 \mathrm{~mL} \mathrm{~min}^{-1}$. The oven temperature was maintained at $60{ }^{\circ} \mathrm{C}$ for $2 \mathrm{~min}$, then ramped to $320^{\circ} \mathrm{C}$ at a rate of $5{ }^{\circ} \mathrm{C} \mathrm{min}{ }^{-1}$, and held for $11 \mathrm{~min}$. Mass spectra collected in the electron impact mode with $70 \mathrm{eV}$ ionization energy were recorded at $0.15 \mathrm{scans} \mathrm{s}^{-1}$, at a scanning range of 44 to $620 \mathrm{~m} / \mathrm{z}$. The resultant data were processed with TurboMass software

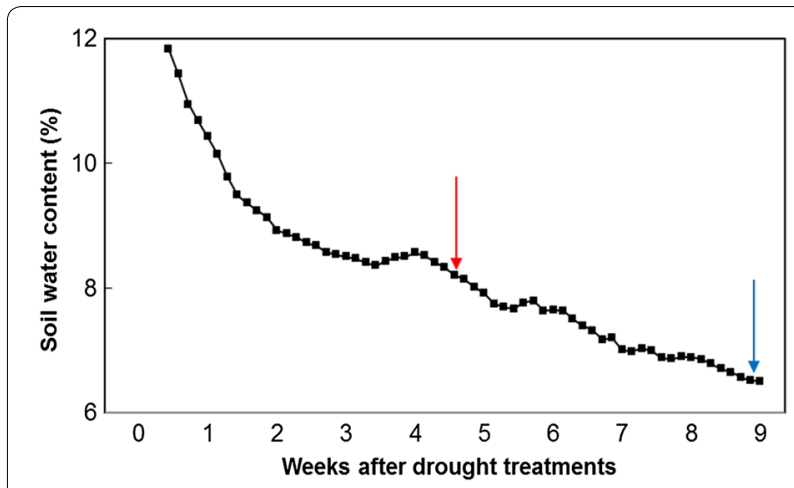

Fig. 1 Soil water content (\%: 10-cm depth) over time after stress treatment. Red arrows indicate times of mild drought; blue arrow, moderate drought 
(version 5.4.2.1617; Perkin-Elmer, Shelton, CT, USA). For the metabolite identification, peaks were compared with retention indices and mass spectra of customized reference mass spectral library (NIST, Gaithersburg, MD, USA) and authentic reference standards. Relative quantification was estimated based on peak area ratio of analyte to internal standard ribitol. All chemicals and reagents were of analytical grade and were not further purified.

\section{LC-MS/MS analysis and data processing}

For LC-MS/MS analysis, the metabolites were extracted as described by de Vos et al. [21], with some modifications. One $\mathrm{mL}$ of methanol/water (8:2, v:v) was added to $100 \mathrm{mg}$ of freeze-dried leaf tissue. The mixture was extracted in an ultra-sonic water bath for $15 \mathrm{~min}$ at $20^{\circ} \mathrm{C}$ and centrifuged at $20,000 \mathrm{~g}$ for $10 \mathrm{~min}$ at room temperature. The supernatant was then passed through a $0.2 \mu \mathrm{m}$ pore-size syringe filter and transferred into a sample vial.

An Ultimate 3000 nano RSLC system (Thermo Fisher Scientific, Waltham, MA, USA) coupled to a Triple TOF 5600 MS system (AB Sciex, Concord, ON, Canada) was used to perform the LC-MS/MS analyses. The LC separation was conducted on a Kinetex C18 100A column $(100 \times 2.1 \mathrm{~mm}$ i.d., $7 \mu \mathrm{m}$ particle size; Phenomenex, Torrance, CA, USA) with an injection volume of $2 \mu \mathrm{L}$ and a column temperature of $40{ }^{\circ} \mathrm{C}$. The mobile phases consisted of A ( $0.1 \%$ formic acid in water) and B $(0.1 \%$ formic acid in acetonitrile) at a flow rate of $0.25 \mathrm{~mL} \mathrm{~min}^{-1}$. Elution was programmed as a linear gradient that began by increasing from 5 to $100 \%$ B in 17 min, held at $100 \%$ B for $3 \mathrm{~min}$, decreasing from 100 to $5 \% \mathrm{~B}$ in $1 \mathrm{~min}$, and then maintained at $5 \%$ B for $2 \mathrm{~min}$. Mass spectra were generated in the positive ion mode with an electrospray ionization (ESI) source. Nitrogen was used as the drying gas at $500{ }^{\circ} \mathrm{C}$ and the ESI spray voltage was $5500 \mathrm{~V}$. Full-scan mass spectra were acquired over an $\mathrm{m} / \mathrm{z}$ range of 50 to 2000.

The resultant data were collected with Analyst TF 1.7 software and feature-finding was performed with Elements software (version 1.3.1; Protemone Software Inc., Portland, OR, USA). Fragment mass tolerance was $0.5 \mathrm{Da}$ for low-resolution and $20 \mathrm{ppm}$ for high-resolution spectra. To identify the metabolites, we utilized various biochemical databases and standard libraries including MassBank of North America (MoNA; http://mona. fiehnlab.ucdavis.edu/), DrugBank (http://www.drugbank. $\mathrm{ca} /$ ), the Yeast Metabolome Database (YMDB; http:// www.ymdb.ca/), Human Metabolome Database (HMDB; http://www.hmdb.ca/), Toxin-Toxin-Target Database (T3DB; http://www.t3db.ca/), and NIST/EPA/NIH Mass Spectral Library (NIST 14). Relative quantification was estimated by the ratio of the respective peak areas to the total peak areas. All solvents were of analytical grade and were used without further purification.

\section{Statistical analysis}

The resultant datasets from both the GC-MS and the LC-MS/MS procedures were subjected to multivariate statistical analysis, using SIMCA-P + software (version 12.0; Umetrics AB, Kinnelon, NJ, USA). All data were Pareto-scaled and an unsupervised principal component analysis (PCA) was conducted to explore dataset variations. For individual metabolites, Student's $t$-tests were performed using STATISTICA (version 8.0; StatSoft Inc., Tulsa, OK, USA) to evaluate the significance of differences $(p<0.05)$ in metabolite levels between transgenic and non-transgenic soybean plants.

\section{Results}

Global metabolite profiling based on GC-MS analysis of soybean plants under drought stress

To examine the global metabolic responses to drought stress by transgenic soybean plants that over-express $A B F 3$, we used an untargeted approach to compare the metabolite profiles between transgenic and nontransgenic lines. A total of 64 metabolites, including sugars, sugar alcohols, amino acids, organic acids, and fatty acids, were identified based on GC-MS platforms from leaf extracts obtained during the treatment period (Table 1, Additional file 1: Fig. S1.). The PCA score plot displayed three clusters that corresponded to different degrees of stress (Fig. 2a). Our PCA loading plot demonstrated that fructose and an unknown sugar mainly influenced the separation by PC 1 (72.3\% of the total variance), while fructose and sucrose contributed to the separation by PC 2 (13.0\% of the total variance) (Fig. 2b). We also observed minor contributions by glucose, melibiose, proline, D-pinitol, glycerol, and $\gamma$-aminobutyric acid.

\section{LC-MS/MS-based metabolic profiling in soybean plants exposed to different drought conditions}

Our LC-MS/MS analysis identified 476 metabolites, including flavonoids, isoflavonoids, phenylpropanoids, steroids, alkaloids, benzenoids, lipids, and amines, in leaves from drought-stressed plants (data not shown). Results from the PCA score plot indicated a significant separation among different degrees of drought stress, but no distinctive clustering in metabolites was detected between transgenic and non-transgenic soybeans (Fig. 3a). The loading plot revealed that the discrimination of samples by PC 1 (35.1\% of the total variance) was mainly due to 9-OxoOTrE and monolinolenin, whereas 6"-malonylgenistin, dehydrophytosphingosine, apiosylglucosyl 4-hydroxybenzoate, and campestanol contributed to the separation of samples by PC 2 (16.4\% of the 
Table 1 Chromatographic and spectrometric data for 64 metabolites from soybean leaves analyzed by GC-MS

\begin{tabular}{|c|c|c|c|c|c|}
\hline Compound & RT & Compound & RT & Compound & RT \\
\hline Oxalic acid & 6.80 & Proline & 19.42 & Xylitol & 27.26 \\
\hline $\begin{array}{l}\text { Propanoic acid, 2-(methoxy- } \\
\text { imino) }\end{array}$ & 8.81 & Ribose & 19.60 & Shikimic acid & 33.49 \\
\hline Uracil & 9.03 & Isoleucine & 19.77 & 3-Hydroxy hexanedioic acid & 34.19 \\
\hline Glutamic acid & 9.63 & Succinic acid & 20.31 & Ribitol & 35.71 \\
\hline Glyceric acid & 12.78 & Isocitric acid & 20.67 & Galactose & 36.39 \\
\hline Ketomalonic acid & 13.85 & beta-Alanine & 21.05 & Inositol & 36.61 \\
\hline Alanine & 14.55 & Fumaric acid & 21.16 & Tyrosine & 37.41 \\
\hline Lactic acid & 14.91 & Glycine & 21.20 & Glucose & 37.66 \\
\hline Xylose & 15.41 & Threonine & 21.26 & Fructose & 38.70 \\
\hline Glycolic acid & 16.18 & 4-Keto glucose & 23.01 & Melibiose & 38.75 \\
\hline Leucine & 17.15 & Threitol & 23.11 & Tryptophane & 39.05 \\
\hline Asparagine & 17.27 & Malic acid & 24.13 & Sorbitol & 40.16 \\
\hline Ribonic acid & 17.46 & Salicylic acid & 24.14 & Glyceryl glycoside & 41.40 \\
\hline Malonic acid & 17.59 & Citric acid & 24.43 & Sucrose & 45.78 \\
\hline Valine & 17.86 & Y-Aminobutyric acid & 24.50 & Maltitol & 45.87 \\
\hline Carboxyglycine & 18.14 & Pyroglutamic acid & 25.10 & Trehalose & 46.59 \\
\hline Hedracylic acid & 18.23 & Threonic acid & 25.66 & Octadecanoic acid & 47.13 \\
\hline Aminoethanol & 18.62 & Phenylalanine & 25.89 & Hexadecanoic acid & 47.99 \\
\hline Lysine & 18.89 & Glutamine & 25.93 & Maltose & 53.91 \\
\hline Mannitol & 18.89 & D-pinitol & 26.55 & Raffinose & 54.59 \\
\hline Serine & 18.94 & Aspartic acid & 26.88 & Lactose & 55.96 \\
\hline Glycerol & 19.20 & Erythritol & 26.93 & & \\
\hline
\end{tabular}

$R T$ retention time (min)

total variance) (Fig. 3b). Glycerol 1-hexadecanoate, flavidulol $\mathrm{C}$, and nervonoylacetone were also contributed to the distinction by $\mathrm{PC} 1$ or $\mathrm{PC} 2$.

\section{Variations between transgenic and non-transgenic soybean plants in primary metabolites obtained from GC- MS data}

We performed relative quantification of identified compounds to examine the significance of differences between transgenic and non-transgenic soybean plants during the treatment period. In the absence of drought stress (Week 0), the relative levels of ketomalonic acid, carboxyglycine, serine, phenylalanine, octadecanoic acid, and lactose were significantly higher in the transgenic line than in the WT, while those of ribonic acid, ribose, 4-keto glucose, salicylic acid, and shikimic acid were significantly lower in the transgenics (Additional file 1: Table S1).

At Week 4 (mild-drought), the relative level of fructose was significantly greater in the transgenic line than in the WT, whereas the relative levels of carboxyglycine and raffinose were significantly lower in the transgenics (Additional file 1: Table S2).
At Week 9 (moderate-drought), the relative levels of leucine, asparagine, valine, lysine, serine, isoleucine, beta-alanine, glycine, threonine, pyroglutamic acid, phenylalanine, 3-hydroxyhexanedioic acid, inositol, and tyrosine were considerably greater in the transgenic line than in the WT (Table 2). In contrast, the relative levels of 4-ketol glucose, threonic acid, and D-pinitol were lower in those transgenics. In particular, the relative levels of free amino acids such as leucine, asparagine, valine, lysine, serine, isoleucine, beta-alanine, glycine, threonine, pyroglutamic acid, phenylalanine, and tyrosine were $40.4 \%, 151.9 \%, 57.5 \%, 126.6 \%, 89.3 \%$, $48.1 \%, 130.0 \%$, $72.2 \%, 80.4 \%, 84.2 \%, 83.8 \%$, and $73.1 \%$ higher, respectively, in the transgenic line than in the WT.

\section{Differences between genotypes in primary and secondary metabolites derived from LC-MS/MS data}

Quantitative comparisons were made between genotypes concerning the metabolites obtained from LC-MS/MS data. Under non-drought conditions (Week 0), relative levels of corchorifatty acid $D$, corchorifatty acid A, PC (16:0/16:0), polyethylene, oxidized, 3,4-methylenesebacic acid, rubraflavone $\mathrm{D}$, neryl arabinofuranosyl-glucoside, $\quad \mathrm{di}(\mathrm{N}$-acetyl-D-glucosamine $)$, 


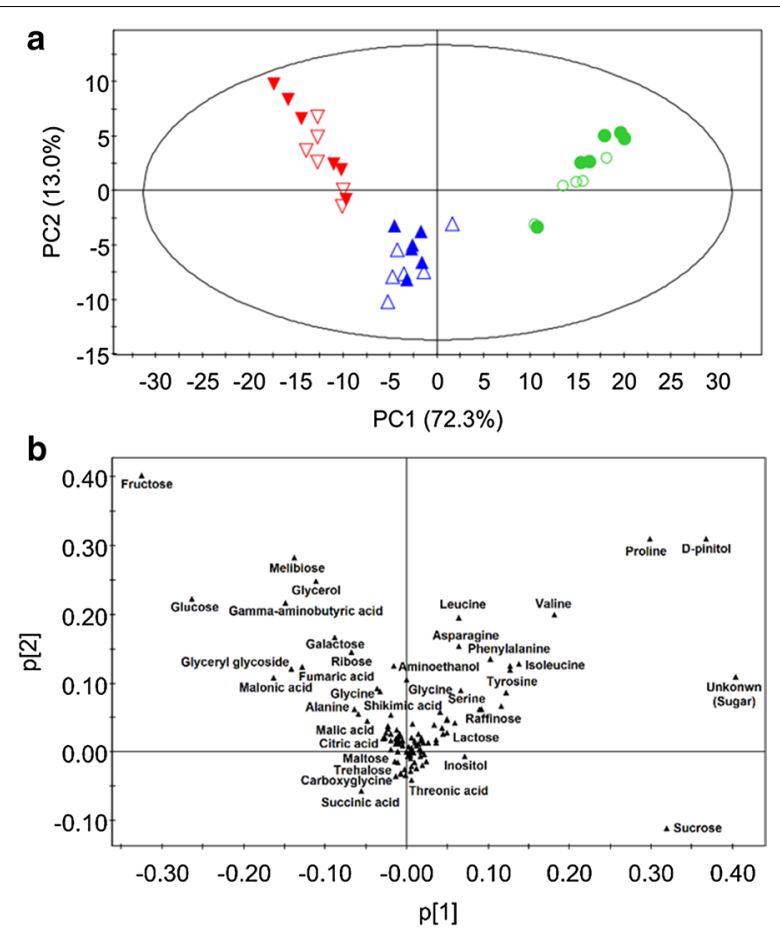

Fig. 2 PCA derived from GC-MS data for drought-tolerant transgenic soybean 'ABF3' (filled symbols) and 'Kwangan'WT (open symbols) grown under non-drought (blue open triangle, blue filled triangle), mild-drought (red inverted open triangle, red inverted filled triangle), and moderate-drought (green open circle, green filled circle) conditions. a Score plot (ellipse represents Hotelling $\mathrm{T}^{2}$ with 95\% confidence) and $\mathbf{b}$ loading plot

3-methyl-3-butenylapiosyl-(1->6)-glucoside, dihydrozeatin-O-glucoside, nequinate, 4-\{[5-(cyclohexylamino $[1,2,4]$ triazolo [1,5-a] pyrimidin-7-yl] amino\} benzenesulfonamide, dihydro-3-(2-octenyl)-2,5-furandione, 3-cis-hydroxy-b,e-caroten-3'-one, 2-amino 1-phenylethanol, DL-phenylalanine, D-tyrosine, ferulic acid, chrysoeriol 7-O-(6"-malonyl-glucoside), and methylpyridazine piperidine butyloxyphenylethylacetatewere significantly higher in the transgenic line than in the WT. In contrast, the levels of DG (14:0/18:1(9Z)/0:0), 4-(3,14-dihydroxy-10,13-dimethyl-hexadecahydrocyclopenta[a] phenanthren-17-Yl)-5h-furan-2-one, delavirdine, ethyl 7-epi-12-ydroxyjasmonate glucoside, adenosine, 2-phenoxyethyl isobutyrate, taraxasterone, PG (18:3(9Z,12Z,15Z)/22:6 (4Z,7Z,10Z,13Z,16Z,19Z)), rheidin C, apo-10'-violaxanthal, momordicoside I, N6-carbamoyl-L-threonyladenosine, and zinc acetate were considerately lower in the transgenics (Additional file 1: Table S3).

In response to mild drought (Week 4), the relative levels of corchorifatty acid A, ricinoleic acid methyl ester, Ser-Leu, 8-oxohexadecanoic acid, D-proline, trigonelline, 5(6)-pentyl-1,4-dioxan-2-one, 4,7(11)-guaiadien-8-one, and 5-megastigmen-7-yne-3,9-diol 3-glucoside were greater in the transgenic line than in the WT, while those of 1-(10Z-heptadecenoyl)-sn-glycero-3-phospho(1'-myo-inositol), formononetin, DG (22:5(7Z,10Z,13Z, 16Z,19Z)/14:0/0:0), and DG (16:1(9Z)/18:1(9Z)/0:0) were lower in the transgenic line (Additional file 1: Table S4).

Under moderate drought (Week 9), the relative levels of trigonelline, beta-D-glucopyranosyl-11-hydroxyjasmonic acid, enol-phenylpyruvate, N-(1-deoxy-1-fructosyl) tyrosine, 3-methyl-3-butenyl apiosyl-(1->6)-glucoside, benzyl O-[arabinofuranosyl-(1->6)-glucoside], dihydrozeatin-O-glucoside, nequinate, 5-aminoimidazole ribonucleotide, 2,5-pyridinedicarboxylic acid, dipropyl ester, D-tyrosine, dihydroxyfumitremorgin $C$, and cingerglycolipid B were higher in the transgenic line than in the WT. In contrast, the relative levels of DG (14:0/18:0/0:0), PI (20:0/20:3(8Z,11Z,14Z)), PI (20:3(5Z,8Z,11Z)/18:0), PI (18:1(11Z)/18:1(9Z)), PI (18:1(9Z)/20:4(8Z,11Z,14 $\mathrm{Z}, 17 \mathrm{Z})$ ), rubraflavone $\mathrm{D}$, captopril-cysteine disulfide, gluten exorphin B5, capsianoside VI, rheidin C, $\mathrm{N}^{\prime}$-hydroxyneosaxitoxin, brassica napus non-fluorescent chlorophyll catabolite 3, aromadendrin, 9-deazainosine, 4-methylthio-alpha-D-mannose, and malformin were lower in those transgenics (Table 3).

\section{Discussion}

Our GC-MS-based metabolic analysis showed clear distinctions among soluble sugar profiles in parallel with the severity of drought treatment. Changes in the amounts of soluble sugars in plants under abiotic stress are associated with the regulation of $\mathrm{CO}_{2}$ assimilation, source-sink carbon partitioning, activity of related enzymes, and the expression of specific genes [22]. Vassey and Sharkey [23] have suggested that the activity of sucrose phosphate synthase, a key enzyme in sucrose synthesis, declines in Phaseolus vulgaris during periods of mild water stress because the photosynthetic rate is reduced due to stomatal restriction of the $\mathrm{CO}_{2}$ supply. Application of exogenous $\mathrm{ABA}$ induces a significant increase in sugar accumulations under drought stress, which may be partly responsible for improving the relative water content in leaves of Oryza sativa [24]. Results provided here demonstrated that drought strongly contributed to the variation in sugar profiles. However, overexpression of $A B F 3$, which confers improved tolerance to drought stress, did not have a significant impact on sugar metabolism in our drought-tolerant transgenic soybeans.

Regulation of phytohormone-related genes in plants is correlated with alterations in various biochemical pathways as well as in primary metabolism. For example, transgenic overexpression of the gene for jasmonic acid carboxyl methyltransferase (AtJMT) increases isoflavone contents in transgenic soybean seeds, which implies that 

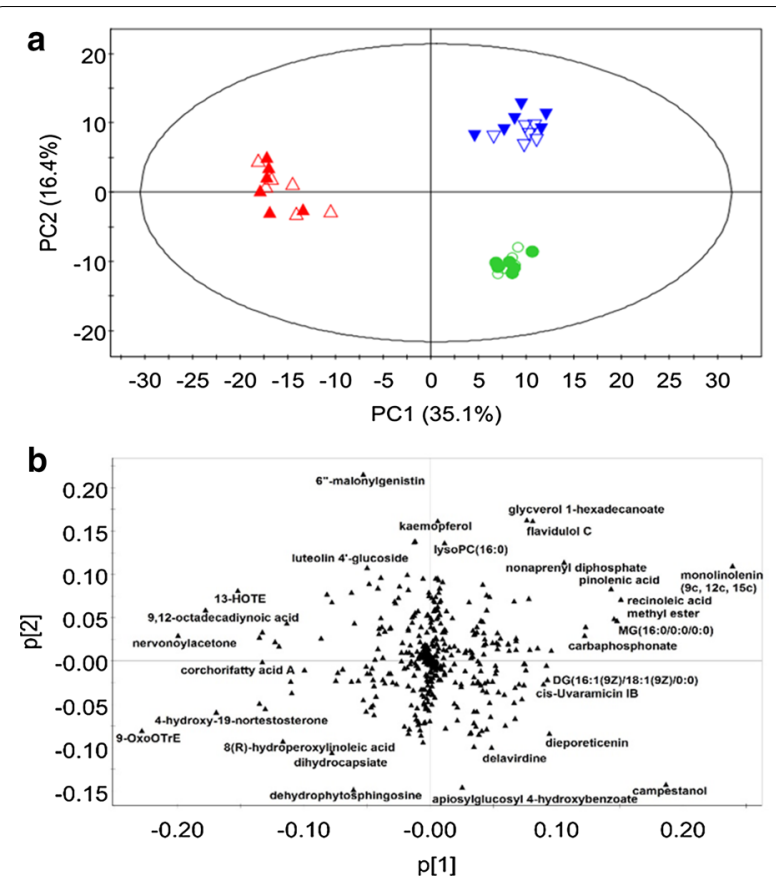

Fig. 3 PCA derived from LC-MS/MS data for drought-tolerant transgenic soybean 'ABF3' (filled symbols) and 'Kwangan'WT (open symbols) grown under non-drought (blue open triangle, blue filled triangle), mild-drought (red inverted open triangle, Red inverted filled triangle), and moderate-drought (green open circle, green filled circle) conditions. a Score plot (ellipse represents Hotelling $\mathrm{T}^{2}$ with 95\% confidence) and b loading plot this overexpression is sufficient to activate the defense system that then promotes isoflavone biosynthesis [25]. A metabolomic approach using LC-MS allows researchers to detect a wide range of analytes-not only primary metabolites but also secondary metabolites such as flavonoids, glucosinolates, phenylpropanoids, phenolic acids, polyamines, saponins, and derivatives [21]. Here, we identified 476 metabolites, both primary and secondary. The PCA indicated that the metabolite profiles could be substantially discriminated among three different degrees of drought stress. However, the separation in metabolites was insignificant between transgenic and non-transgenic soybeans, an outcome that corresponded to the result derived from GC-MS data. These findings suggest that overexpression of $A B F 3$ in transgenic soybean might lead to a negligible difference in primary and secondary metabolism when compared with its non-transgenic counterpart when both are grown under drought conditions.

With regard to metabolic variations, environmental factors, e.g., growing season, location, sowing date, and type of abiotic stress, play a larger role than genetic modifications [26-28]. Because those factors may be the dominant parameter driving the variability of metabolite profiles in the PCA, we also conducted a comparative analysis to examine individual differences in the datasets of transgenic soybean versus its non-transgenic counterpart. Relative quantification demonstrated that some metabolites were accumulated due to overexpression

Table 2 Relative quantification of GC-MS-based metabolites in 'Kwangan' WT soybean and drought-tolerant transgenic soybean 'ABF3' measured after 9 weeks of moderate-drought treatment

\begin{tabular}{|c|c|c|c|c|c|}
\hline Component & Kwangan & ABF3 & $t$-value & Degrees of freedom & $p$ value \\
\hline Leucine & $3.89 \pm 0.79$ & $5.46 \pm 1.41$ & -2.38 & 10 & 0.039 \\
\hline Asparagine & $1.49 \pm 0.55$ & $3.75 \pm 1.58$ & -3.30 & 10 & 0.008 \\
\hline Valine & $10.4 \pm 3.01$ & $16.3 \pm 4.69$ & -2.61 & 10 & 0.026 \\
\hline Lysine & $0.29 \pm 0.18$ & $0.66 \pm 0.21$ & -3.11 & 9 & 0.013 \\
\hline Serine & $1.58 \pm 0.52$ & $3.00 \pm 1.03$ & -3.02 & 10 & 0.013 \\
\hline Isoleucine & $5.91 \pm 1.21$ & $8.76 \pm 2.14$ & -2.83 & 10 & 0.018 \\
\hline beta-Alanine & $0.66 \pm 0.35$ & $1.51 \pm 0.49$ & -3.49 & 10 & 0.006 \\
\hline Glycine & $0.46 \pm 0.06$ & $0.79 \pm 0.23$ & -3.35 & 10 & 0.007 \\
\hline Threonine & $0.62 \pm 0.17$ & $1.11 \pm 0.24$ & -4.10 & 10 & 0.002 \\
\hline 4-Keto glucose & $0.06 \pm 0.01$ & $0.04 \pm 0.01$ & 3.08 & 10 & 0.012 \\
\hline Pyroglutamic acid & $0.91 \pm 0.17$ & $1.68 \pm 0.40$ & -4.30 & 10 & 0.002 \\
\hline Threonic acid & $0.40 \pm 0.06$ & $0.29 \pm 0.04$ & 3.76 & 10 & 0.004 \\
\hline Phenylalanine & $3.54 \pm 0.90$ & $6.51 \pm 1.85$ & -3.53 & 10 & 0.005 \\
\hline D-Pinitol & $106.3 \pm 9.33$ & $93.5 \pm 3.12$ & 2.95 & 6 & 0.026 \\
\hline 3-Hydroxy hexadioic acid & $0.22 \pm 0.03$ & $0.39 \pm 0.07$ & -5.32 & 10 & $<0.001$ \\
\hline Inositol & $14.3 \pm 1.40$ & $19.9 \pm 3.71$ & -3.46 & 10 & 0.006 \\
\hline Tyrosine & $4.91 \pm 0.93$ & $8.50 \pm 1.43$ & -5.16 & 10 & $<0.001$ \\
\hline
\end{tabular}

Relative amounts were determined by peak area ratio of analyte to internal standard. Values are means $(n=6) \pm$ standard deviations. $p$-values are from Student's t-tests 
Table 3 Relative quantification of LC-MS/MS-based metabolites in 'Kwangan' WT soybean and drought-tolerant transgenic soybean 'ABF3' measured after 9 weeks of moderate-drought treatment

\begin{tabular}{|c|c|c|c|c|c|}
\hline Component & Kwangan & ABF3 & $t$-value & $\begin{array}{l}\text { Degrees } \\
\text { of freedom }\end{array}$ & $p$-value \\
\hline$D G(14: 0 / 18: 0 / 0: 0)$ & $5.44 \pm 0.03$ & $5.31 \pm 0.06$ & 4.96 & 10 & 0.001 \\
\hline Trigonelline & $5.64 \pm 0.08$ & $5.77 \pm 0.07$ & -3.18 & 10 & 0.010 \\
\hline PI(20:0/20:3(8Z,11Z,14Z)) & $5.14 \pm 0.09$ & $5.01 \pm 0.10$ & 2.43 & 10 & 0.035 \\
\hline $\mathrm{PI}(20: 3(5 Z, 8 Z, 11 Z) / 18: 0)$ & $5.58 \pm 0.09$ & $5.40 \pm 0.05$ & 4.04 & 9 & 0.003 \\
\hline beta-D-Glucopyranosyl-1 1-hydroxyjasmonic acid & $5.92 \pm 0.61$ & $6.62 \pm 0.28$ & -2.57 & 10 & 0.028 \\
\hline $\mathrm{PI}(18: 1(11 Z) / 18: 1(9 Z))$ & $4.44 \pm 0.46$ & $3.97 \pm 0.23$ & 2.25 & 10 & 0.048 \\
\hline PI(18:1 (9Z)/20:4(8Z,11Z,14Z,17Z)) & $4.44 \pm 046$ & $3.97 \pm 0.23$ & 2.25 & 10 & 0.048 \\
\hline Enol-phenylpyruvate & $4.52 \pm 0.11$ & $4.78 \pm 0.07$ & -4.67 & 10 & 0.001 \\
\hline Rubraflavone D & $4.04 \pm 0.14$ & $3.70 \pm 0.34$ & 2.28 & 10 & 0.046 \\
\hline Captopril-cysteine disulfide & $5.04 \pm 0.09$ & $4.76 \pm 0.10$ & 5.03 & 10 & 0.001 \\
\hline Gluten exorphin B5 & $4.85 \pm 0.19$ & $3.91 \pm 0.62$ & 3.26 & 9 & 0.010 \\
\hline Capsianoside VI & $5.61 \pm 0.04$ & $5.22 \pm 0.32$ & 2.84 & 6 & 0.029 \\
\hline Rheidin C & $4.54 \pm 0.31$ & $3.59 \pm 0.14$ & 4.09 & 6 & 0.006 \\
\hline N-(1-Deoxy-1-fructosyl)tyrosine & $5.03 \pm 0.14$ & $5.20 \pm 0.10$ & -2.48 & 10 & 0.032 \\
\hline 3-Methyl-3-butenyl apiosyl-(1->6)-glucoside & $3.77 \pm 0.80$ & $4.71 \pm 0.16$ & -2.88 & 8 & 0.020 \\
\hline Benzyl O-[arabinofuranosyl-(1->6)-glucoside] & $3.58 \pm 0.67$ & $4.42 \pm 0.16$ & -3.02 & 8 & 0.017 \\
\hline Veranisatin C & $5.25 \pm 0.07$ & $5.12 \pm 0.05$ & 4.07 & 10 & 0.002 \\
\hline N'-Hydroxyneosaxitoxin & $4.21 \pm 0.38$ & $3.53 \pm 0.34$ & 3.11 & 9 & 0.012 \\
\hline Dihydrozeatin-O-glucoside & $3.35 \pm 0.38$ & $4.25 \pm 0.39$ & -3.62 & 8 & 0.007 \\
\hline Nequinate & $3.35 \pm 0.38$ & $4.25 \pm 0.39$ & -3.62 & 8 & 0.007 \\
\hline Brassica napus non-fluorescent chlorophyll catabolite 3 & $8.09 \pm 0.87$ & $4.41 \pm 1.69$ & 4.33 & 8 & 0.002 \\
\hline 5-Aminoimidazole ribonucleotide & $4.89 \pm 0.42$ & $5.35 \pm 0.24$ & -2.32 & 10 & 0.043 \\
\hline Aromadendrin & $4.41 \pm 0.28$ & $3.86 \pm 0.52$ & 2.32 & 10 & 0.043 \\
\hline 9-Deazainosine & $4.28 \pm 0.27$ & $3.79 \pm 0.44$ & 2.30 & 10 & 0.044 \\
\hline 4-Methylthio-Alpha-D-Mannose & $5.17 \pm 0.07$ & $5.00 \pm 0.04$ & 5.54 & 10 & $<0.001$ \\
\hline Malformin & $4.92 \pm 0.33$ & $4.32 \pm 0.39$ & 2.86 & 10 & 0.017 \\
\hline 2,5-Pyridinedicarboxylic acid, dipropyl ester & $4.61 \pm 0.30$ & $5.05 \pm 0.21$ & -2.93 & 10 & 0.015 \\
\hline D-Tyrosine & $5.45 \pm 0.06$ & $5.61 \pm 0.04$ & -5.10 & 10 & $<0.001$ \\
\hline Dihydroxyfumitremorgin C & $4.65 \pm 0.11$ & $4.88 \pm 0.09$ & -4.17 & 10 & 0.002 \\
\hline Gingerglycolipid B & $4.73 \pm 0.24$ & $5.00 \pm 0.12$ & -2.36 & 9 & 0.042 \\
\hline
\end{tabular}

Relative amounts were determined the ratio of the respective areas to total peak areas. Values are means $(\mathrm{n}=6) \pm s t a n d a r d$ deviations. $p$-values are from Student's $t$-tests

of $A B F 3$ during the stress period. In particular, levels of amino acids were somewhat higher in our tolerant transgenic soybean than in the non-transgenics in response to moderate drought. Proline participates in defenses against osmotic challenges by acting as a major compatible solute [29]. Yoshiba et al. [30] have suggested that ABA is involved in activating the gene that encodes $\Delta^{1}$ pyrroline-5-carboxylate synthase, the key enzyme for proline biosynthesis.

Inhibition of ABA accumulations, as studied with the ABA-deficient Zea mays (maize) mutant vp5, substantially decreases proline concentrations in primary roots exposed to low soil water potential [31]. However, Verslues and Bray [32] have reported that an ABA accumulation is required for proline accumulation, and that $\mathrm{ABA}$ alone is not sufficient to elicit the accumulation of proline at low water potential. Our results indicated that proline levels were considerably elevated under moderate-drought stress in both transgenic and non-transgenic plants, but that those levels were not significantly different between genotypes. In addition, asparagine, beta-alanine, glycine, isoleucine, leucine, lysine, phenylalanine, pyroglutamic acid, serine, threonine, tyrosine, and valine were accumulated at higher levels in the transgenics than in the WT under moderatedrought stress. Rai and Sharama [33] have reported that amino acids modulate stomatal closure, stomatal resistance, and $\mathrm{K}^{+}$fluxes in the guard cells of Vicia faba leaves. 
However, this ABA-induced stomatal closure is reversed by amino acids that promote stomatal opening, and is further enhanced by glycine, alanine, leucine, threonine, lysine, arginine, proline, tryptophan, and phenylalanine [33]. That research group has suggested that this behavior could be a balancing mechanism by which an optimal stomatal aperture is maintained under stress because ABA-induced stomatal closure under drought conditions reduces the loss of water but can have detrimental effects on gas exchange. Finally, an ABA-deficient mutant of Arabidopsis thaliana accumulates a smaller amount of free amino acids during dehydration when compared with the WT [34].

The accumulation of free amino acids may also serve as a precursor step in the biosynthesis of endogenous antioxidants and plant hormones that are involved in the response to osmotic stress as well as substrates for the synthesis of stress-induced proteins [35]. Moreover, ABA-enhanced tolerance to water stress is correlated with the activation of antioxidant defense systems by that hormone [36]. Therefore, the elevated levels of amino acids in our $A B F 3$-overexpressing transgenics might be explained by the closing of stomata and various successive biochemical reactions as part of a drought defense mechanism.

A recent multi-omics study with soybean leaves has revealed that exogenously applied ABA increases the accumulation of glycerolipids, prenol lipids, and phospholipids while the concentrations of secondary metabolites, such as flavonoids and isoflavonoids, remain unchanged [37]. Drought stress is known to have a distinct effect on the metabolism of lipids that play a role in maintaining membrane integrity and preserving cell compartmentation $[38,39]$. Overexpression of the gene for phosphatidylinositol synthase, ZmPIS, in maize plants effectively improves their drought tolerance by altering the lipid composition in membranes and increasing ABA synthesis [40]. Here, overexpression of $A B F 3$ in soybean plants changed the levels of some glycerolipids, glycerophospholipids, prenol lipids, fatty acyls, and their derivatives during the stress period. These alterations might have been correlated with the regulation of cell membrane damage and the osmotic balance in response to drought. Nevertheless, quantitative differences in the relative levels of those metabolites were less than $50 \%$ between genotypes.

In conclusion, the induction of drought stress significantly altered the accumulations of primary and secondary metabolites, as indicated by our GC-MS and LC-MS/ MS analyses. However, no changes in metabolite profiles were apparent between the transgenic and non-transgenic soybeans. Under drought conditions, only a slight difference in some metabolites was detected between transgenic soybean and the WT. These findings suggest that the overexpression of $A B F 3$ in soybean might lead to an insignificant difference in primary and secondary metabolism when compared with its non-transgenic counterpart after drought treatment. Further studies that examine the effect of the interactions between waterstress conditions and genotype in metabolite profiles will provide better information about the variability in drought-related metabolic responses that result from this genetic transformation.

\section{Additional file}

\begin{abstract}
Additional file 1: Table S1. Relative quantification of GC-MS-based metabolites in 'Kwangan'WT soybean and drought-tolerant transgenic soybean 'ABF3' measured at beginning of stress treatment (Week 0; nondrought). Table S2. Relative quantification of GC-MS-based metabolites in 'Kwangan'WT soybean and drought-tolerant transgenic soybean 'ABF3' measured after 4 weeks of mild-drought treatment. Table S3. Relative quantification of LC-MS/MS-based metabolites in 'Kwangan'WT soybean and drought-tolerant transgenic soybean 'ABF3' measured at beginning of stress treatment (Week 0: non-drought). Table S4. Relative quantification of LC-MS/MS-based metabolites in 'Kwangan'WT soybean and droughttolerant transgenic soybean 'ABF3' measured after 4 weeks of milddrought treatment. Figure S1. Representative GC-MS chromatographic profile of soybean grown under non-drought (A), mild-drought (B), and moderate-drought (C) conditions.
\end{abstract}

\section{Authors' contributions}

KHN and CGK designed the experiment and analyzed the data. HJK, YSC, and SYK contributed to the plant material preparation. DYK, HJK, and ISP per-

formed the experiments. KHN wrote the paper. All authors read and approved the final manuscript.

\section{Author details}

${ }^{1}$ Division of Ecological Conservation, National Institute of Ecology, Seocheon 33657, Republic of Korea. ${ }^{2}$ Bio-Evaluation Center, Korea Research Institute of Bioscience \& Biotechnology, Cheongju 28116, Republic of Korea. ${ }^{3}$ Department of Genetic Engineering, Dong-A University, Busan 49315, Republic of Korea. ${ }^{4}$ Department of Biotechnology, Chonnam National University, Gwangju 61186, Republic of Korea.

\section{Acknowledgements}

This research was supported by Grants from the KRIBB Research Initiative Program, the Next-Generation BioGreen 21 Program (No. PJ01368601), Rural Development Administration, and the National Institute of Ecology (NIE-A2019-04), Ministry of Environment, Republic of Korea.

\section{Competing interests}

The authors declare that they have no competing interests.

\section{Publisher's Note}

Springer Nature remains neutral with regard to jurisdictional claims in published maps and institutional affiliations.

Received: 14 October 2018 Accepted: 20 November 2018 Published online: 20 March 2019 


\section{References}

1. Zeevaart JAD, Creelman RA (1988) Metabolism and physiology of abscisic acid. Annu Rev Plant Physiol Plant Mol Biol 39:439-473

2. Leung J, Giraudat J (1998) Abscisic acid signal transduction. Annu Rev Plant Physiol Plant Mol Biol 49:199-222

3. Roelfsema MRG, Prins HBA (1995) Effect of abscisic acid on stomatal opening in isolated epidermal strips of abi mutants of Arabidopsis thaliana. Physiol Plant 95:373-378

4. Xu XX, Shao HB, Ma YY, Xu G, Sun JN, Guo DG, Ruan CJ (2010) Biotechnological implications from abscisic acid (ABA) roles in cold stress and leaf senescence as an important signal for improving plant sustainable survival under abiotic-stressed conditions. Crit Rev Biotechnol 30:222-230

5. Choi H, Hong J, Ha J, Kang J, Kim SY (2000) ABFs, a family of ABA-responsive element binding factors. J Biol Chem 275:1723-1730

6. Uno Y, Furihata T, Abe H, Yoshida R, Shinozaki K, Yamaguchi-Shinozaki K (2000) Arabidopsis basic leucine zipper transcription factors involved in an abscisic acid-dependent signal transduction pathway under drought and high-salinity conditions. Proc Natl Acad Sci USA 97:11632-11637

7. Kang J, Choi H, Im M, Kim SY (2002) Arabidopsis basic leucine zipper proteins that mediate stress-responsive abscisic acid signaling. Plant Cell 14:343-357

8. Oh SJ, Song SI, Kim YS, Jang HJ, Kim SY, Kim M, Kim YK, Nahm BH, Kim JK (2005) Arabidopsis CBF3/DREB1A and ABF3 in transgenic rice increased tolerance to abiotic stress without stunting growth. Plant Physiol 138:341-351

9. Vanjildorj E, Bae TW, Riu KZ, Kim SY, Lee HY (2005) Overexpression of Arabidopsis ABF3 gene enhances tolerance to drought and cold in transgenic lettuce (Lactuca sativa). Plant Cell Tiss Org Cult 83:41-50

10. Abdeen A, Schnell J, Miki B (2010) Transcriptome analysis reveals absence of unintended effects in drought-tolerant transgenic plants overexpressing the transcription factor ABF3. BMC Genom 11:69

11. Kim HJ, Cho HS, Park JH, Kwon T, Lee JH, Kim DH, Lee DH, Kim CG, Chung YS (2018) Confirmation of drought tolerance of $A B F 3$ gene in soybean. Mol Cells 41:413-422

12. Choi YS, Kim YM, Hwang OJ, Han YJ, Kim SY, Kim JI (2013) Overexpression of Arabidopsis ABF3 gene confers enhanced tolerance to drought and heat stress in creeping bentgrass. Plant Biotechnol Rep 7:165-173

13. Manetti C, Bianchetti C, Casciani L, Castro C, Di Cocco ME, Miccheli A, Motto M, Conti F (2006) A metabonomic study of transgenic maize (Zea mays) seeds revealed variations in osmolytes and branched amino acids. J Exp Bot 57:2613-2625

14. Merewitz EB, Du H, Yu W, Liu Y, Gianfagna T, Huang B (2012) Elevated cytokinin content in ipt transgenic creeping bentgrass promotes drought tolerance through regulating metabolite accumulation. J Exp Bot 63:1315-1328

15. Jiao Z, Si X, Li G, Zhang Z, Xu X (2010) Unintended compositional changes in transgenic rice seeds (Oryza sativa L.) studied by spectral and chromatographic analysis coupled with chemometrics methods. J Agric Food Chem 58:1746-1754

16. Iwaki T, Guo L, Ryals JA, Yasuda S, Shimazaki T, Kikuchi A, Watanabe KN, Kasuga M, Yamaguchi-Shinozaki K, Ogawa T, Ohta D (2013) Metabolic profiling of transgenic potato tubers expressing Arabidopsis dehydration response element-binding protein 1A (DREB1A). J Agric Food Chem 61:893-900

17. Kim JK, Park SY, Lee SM, Lim SH, Kim HJ, Oh SD, Yeo Y, Cho HS, Ha SH (2013) Unintended polar metabolite profiling of carotenoid-biofortified transgenic rice reveals substantial equivalence to its non-transgenic counterpart. Plant Biotechnol Rep 7:121-128

18. James C (2017) Global status of commercialized biotech/GM crops: 2017. ISAAA Briefs No. 53. Ithaca, NY, USA

19. Nam KH, Kim DY, Shin HJ, Nam KJ, An JH, Pack IS, Park JH, Jeong SC, Kim HB, Kim CG (2014) Drought stress-induced compositional changes in tolerant transgenic rice and its wild type. Food Chem 153:145-150

20. Nam KH, Shin HJ, Nam KJ, Pack IS, Park JH, Kim HB, Kim CG (2016) Metabolomic changes in grains of well-watered and drought-stressed transgenic rice. J Sci Food Agric 96:807-814
21. de Vos RC, Moco S, Lommen A, Keurentjes JJB, Bino RJ, Hall RD (2007) Untargeted large-scale plant metabolomics using liquid chromatography coupled to mass spectrometry. Nat Protoc 2:778-791

22. Rosa M, Prado C, Podazza G, Interdonata R, González JA, Hilal M, Prado FE (2009) Soluble sugars-metabolism, sensing and abiotic stress: a complex network in the life of plants. Plant Signal Behav 4:388-393

23. Vassey TL, Sharkey TD (1989) Mild water stress of Phaseolus vulgaris plants leads to reduced starch synthesis and extractable sucrose phosphate synthase activity. Plant Physiol 89:1066-1070

24. Pattanagul W (2011) Exogenous abscisic acid enhances sugar accumulation in rice (Oryza sativa L.) under drought stress. Asian J Plant Sci 10:212-219

25. Nam KH, Kim DY, Pack IS, Park JH, Seo JS, Choi YD, Cheong JJ, Kim CH, Kim CG (2016) Comparative analysis of chemical compositions between nontransgenic soybean seeds and those from plants over-expressing AtJMT, the gene for jasmonic acid carboxyl methyltransferase. Food Chem 196:236-241

26. Chang Y, Zhao C, Zhu Z, Wu Z, Zhou J, Zhao Y, Lu X, Xu G (2012) Metabolic profiling based on LC/MS to evaluate unintended effects of transgenic rice with cry $1 \mathrm{Ac}$ and sck genes. Plant Mol Biol 78:477-487

27. Frank T, Röhlig RM, Davis HV, Barros E, Engel K (2012) Metabolite profiling of maize kernels - genetic modification versus environmental influence. J Agric Food Chem 60:3005-3012

28. Kim MS, Baek SH, Park SU, Im KH, Kim JK (2017) Targeted metabolite profiling to evaluate unintended metabolic changes of genetic modification in resveratrol-enriched rice (Oryza sativa L.). Appl Biol Chem 60:205-214

29. Hare PD, Cress WA (1997) Metabolic implications of stress-induced proline accumulation in plants. Plant Growth Regul 21:79-102

30. Yoshiba Y, Kiyosue T, Katagiri T, Ueda H, Mizoguchi T, Yamaguchi-Shinozak K, Wada K, Harada Y, Shinozaki K (1995) Correlation between the induction of a gene for $\Delta^{1}$-pyrroline-5-carboxylate synthetase and the accumulation of proline in Arabidopsis thaliana under osmotic stress. Plant J 7:751-760

31. Ober ES, Sharp RE (1994) Proline accumulation in maize (Zea mays L.) primary roots at low water potentials. I. Requirement for increased levels of abscisic acid. Plant Physiol 105:981-987

32. Verslues PE, Bray EA (2006) Role of abscisic acid (ABA) and Arabidopsis thaliana $A B A$-insensitive loci in low water potential-induced $A B A$ and proline accumulation. J Exp Bot 57:201-212

33. Rai VK, Sharama UD (1991) Amino acids can modulate ABA induced stomatal closure, stomatal resistance and $\mathrm{K}^{+}$fluxes in Vicia faba leaves. Beitr Biol Pflanz 66:393-405

34. Nambara E, Kawaide H, Kamiya Y, Naito S (1998) Characterization of an Arabidopsis thaliana mutant that has a defect in ABA accumulation: ABA-dependent and ABA-independent accumulation of free amino acids during dehydration. Plant Cell Physiol 39:853-858

35. Rai VK (2002) Role of amino acids in plant responses to stresses. Biol Plant 45:481-487

36. Jiang MY, Zhang JH (2004) Abscisic acid and antioxidant defense in plant cells. Acta Bot Sin 46:1-9

37. Gupta R, Min CW, Kramer K, Agrawal GK, Rakwal R, Park KH, Wang Y, Finkemeier I, Kim ST (2018) A multi-omics analysis of Glycine max leaves reveals alteration in flavonoid and isoflavonoid metabolism upon ethylene and abscisic acid treatment. Proteomics 18:1700366

38. Repellin A, Pham Thi AT, Tashakorie A, Sahsah Y, Daniel C, Zuily-Fodil Y (1997) Leaf membrane lipids and drought tolerance in young coconut palms (Cocos nucifera L.). Eur J Agric 6:25-33

39. Gigon A, Matos AR, Laffray D, Zuily-Fodil Y, Pham-Thi AT (2004) Effect of drought stress on lipid metabolism in the leaves of Arabidopsis thaliana (Ecotype Columbia). Ann Bot 94:345-351

40. Liu X, Zhai S, Zhao Y, Sun B, Liu C, Yang A, Zhang J (2013) Overexpression of the phosphatidylinositol synthase gene (ZmPIS) conferring drought stress tolerance by altering membrane lipid composition and increasing ABA synthesis in maize. Plant, Cell Environ 36:1037-1055 